\section{Efficacy of Molecular Markers Jnurf13 and AcPms1 for Prediction of Genotypes at the Nuclear Ms Locus in Three Open-pollinated Populations of Onion from North America}

\author{
Michael J. Havey ${ }^{1}$ \\ USDA-ARS and Department of Horticulture, University of Wisconsin, \\ Madison, WI 53706 \\ Christopher von Kohn \\ Department of Horticulture, University of Wisconsin, Madison, WI 53706
}

\begin{abstract}
Seed of hybrid onion (Allium cepa L.) is produced using cytoplasmic male sterility (CMS). For the most widely used source of onion CMS, male sterility is conditioned by the interaction of male sterile (S) cytoplasm and the homozygous recessive genotype at the nuclear male fertility locus Ms. Because of the biennial generation time of onion, classical crossing and segregation analyses take years to establish cytoplasms and genotypes at $M s$. Numerous molecular markers have been developed to distinguish onion cytoplasms and estimate genotypes at Ms. Two nuclear markers (jnurf13 and AcPms1) have been reported to cosegregate with $M s$ and correctly predict genotypes in commercial breeding lines and diverse onion germplasm; however, these markers were less predictive for open-pollinated (OP) populations from India. We evaluated the efficacy of jnurf13 and AcPms1 to correctly classify genotypes at $M s$ using 144 random plants from three OP populations of long-day onion from North America. No recombination events were detected between AcPms1 and the Ms locus and three events occurred between jnurf13 and Ms. Our results support either marker as a useful tool to predict genotypes at $M s$ in North American populations of onion, with AcPms1 being the better of the two.
\end{abstract}

Hybrid onion (Allium cepa L.) cultivars are widely grown around the world due in part to hybrid vigor and greater uniformity. Because onion has perfect flowers, production of hybrid seed is based on systems of CMS. For the most widely used CMS in onion, male sterility is conditioned by the interaction of male sterile (S) cytoplasm and the homozygous recessive genotype ( $\mathrm{msms}$ ) at the nuclear male fertility locus $M s$ (Jones and Clarke, 1943). Plants with dominant allele(s) at $M s$ and $\mathrm{S}$ cytoplasm are male fertile. All plants possessing normal $(\mathrm{N})$ male fertile cytoplasm are male fertile regardless of genotypes at $M s$. Because of the biennial generation time of onion, the use of classical crossing to determine cytoplasms

Received for publication 1 June 2017. Accepted for publication 5 July 2017

We gratefully acknowledge the technical help of Christy Stewart.

Names are necessary to report factually on available data; however, the U.S. Department of Agriculture (USDA) neither guarantees nor warrants the standard of the product, and the use of the name by USDA implies no approval of the product to the exclusion of others that may also be suitable.

${ }^{1}$ Corresponding author. E-mail: mjhavey@wisc. edu. and genotypes at $M s$ is a slow process requiring years. Significant effort has been made to identify molecular markers that distinguish onion cytoplasms and predict genotypes at $M s$. S cytoplasm is an alien cytoplasm that was introgressed into onion populations (Havey, 1993), and therefore, many molecular markers in the chloroplast and mitochondrial DNAs confidently distinguish $\mathrm{N}$ and $\mathrm{S}$ cytoplasms (Havey, 1993, 1995; Kim et al., 2009; Sato, 1998; von Kohn et al., 2013). Many molecular markers have also been described that show linkage to the nuclear $M s$ locus (Bang et al., 2013; Gökçe et al., 2002; Havey, 2013; Huo et al., 2012; Kim, 2014; Kim et al., 2015; Park et al., 2013; Yang et al., 2012), even though in some cases few polymorphisms were screened on relatively small numbers of segregating progenies. The plethora of nuclear markers showing linkage to $M s$ can be explained by the location of this locus close to the centromere of chromosome 2 in a region of relatively low recombination nuclear markers may be in linkage disequilibrium with $M s$ and useful to predict genotypes at $M s$, allowing breeders to advance for crossing only plants likely to possess the desired genotype. (Khrustaleva et al., 2016). Nevertheless these
Kim (2014) and Kim et al. (2015) identified two indel markers (jnurf13 and AcPms1, respectively) that cosegregated with $M s$ in a large $F_{2}$ family and consistently predicted genotypes at $M s$ in commercial breeding lines and diverse onion germplasm. The marker AcPms1 resides in the onion homolog of mismatch repair (Msh1), which was proposed as a candidate gene for Ms (Kim et al., 2015). However Khar and Saini (2016) used markers jnurf13 and AcPms1 to genotype random plants from Indian populations of onion and reported that both markers did not always predict correct genotypes at $M s$. In this study, we establish the efficacy of markers jnurf13 and AcPms1 to classify genotypes at $M s$ using random plants from three OP populations of North American onion.

\section{Materials and Methods}

Genotypes at $M s$ were determined for 144 random plants from three OP populations of long-day storage onions ('Brigham Yellow Globe', 'Mountain Danvers', and 'Sapporo-Ki') and DNAs were isolated as previously described (Gökçe et al., 2002). Primers and conditions for the polymerase chain reaction (PCR) for marker jnurf13 were as described by Kim (2014). For marker AcPms1, we obtained inconsistent results using conditions for PCR reported by Kim et al. (2015) and therefore developed a touchdown PCR which more consistently produced amplicons of expected sizes. Reactions consisted of 1× PCR Buffer of the manufacturer (Promega, Fitchburg WI), $2.5 \mathrm{mM} \mathrm{MgCl}_{2}$, $0.25 \mathrm{~mm}$ NTP, 1.25 unit Taq polymerase, and $1 \mu \mathrm{M}$ of primers with $50 \mathrm{ng}$ of onion DNA. Cycling conditions were $95^{\circ} \mathrm{C}$ for $5 \mathrm{~min}$; six cycles of $95^{\circ} \mathrm{C}$ for $30 \mathrm{~s}, 69^{\circ} \mathrm{C}$ for 1 min with a decrease of $2{ }^{\circ} \mathrm{C}$ per cycle, and $72{ }^{\circ} \mathrm{C}$ for $45 \mathrm{~s}$; seven cycles of $95^{\circ} \mathrm{C}$ for $30 \mathrm{~s}, 59^{\circ} \mathrm{C}$ for 1 min with a decrease of $1{ }^{\circ} \mathrm{C}$ per cycle, and $72{ }^{\circ} \mathrm{C}$ for $45 \mathrm{~s}$; and then 27 cycles of $95^{\circ} \mathrm{C}$ for $30 \mathrm{~s}, 52{ }^{\circ} \mathrm{C}$ for $1 \mathrm{~min}$, and $72{ }^{\circ} \mathrm{C}$ for $45 \mathrm{~s}$. Amplicons for both markers were resolved through $8 \%$ acrylamide gels and visualized by silver staining.

\section{Results and Discussion}

The jnurf13 and AcPms1 markers were reported to cosegregate with alleles at $M s$ for a large $\mathrm{F}_{2}$ family and to predict genotypes at $M s$ across diverse onion germplasm (Kim, 2014; Kim et al., 2015). We genotyped these two markers using 144 random plants from three OP populations of long-day onions from North America, which had been previously scored for genotypes at $M s$ (Gökçe et al., 2002) and showed evidence of linkage equilibrium near Ms (Gökçe and Havey, 2002). No recombination events were detected between AcPms1 and the Ms locus for all plants from the three OP populations. We identified three recombination events between jnurf13 and $M s$ (Fig. 1). Kim et al. (2015) reported recombination between jnurf13 and AcPms1 in USDA PI 233186. 


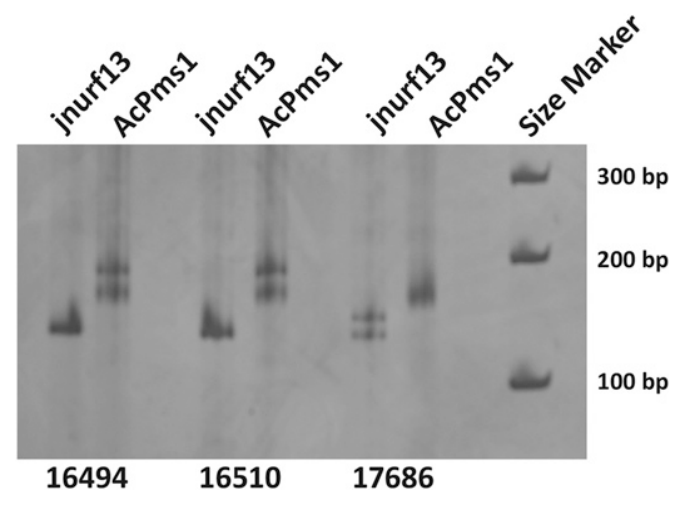

Fig. 1. Recombination detected between molecular markers jnurf13 and AcPms1 revealed by silver staining of amplicons in an 8\% polyacrylamide gel. DNAs were isolated from single plants 16494 and 16510 (both heterozygous at $M s$ ) and 17686 (homozygous dominant at $M s$ ). Sizes of marker in base pairs (bp) is shown on right. Recombination events between the jnurf13 marker and the Ms locus are revealed by the presence of one amplicon for plants 16494 and 16510 and two amplicons for plant 17686 .

These results clearly support either marker as a useful tool to predict genotypes at $M s$, with AcPms1 being the better of the two.

Kim et al. (2015) proposed AcPms1 as a candidate gene for $M s$; however, Khar and Saini (2016) reported that this marker did not accurately predict male fertility restoration for random S-cytoplasmic plants from Indian populations. This inconsistency could be due to rare recombination event(s) between AcPms1 and $M s$, or possibly different male fertility restoration locus (loci) for Scytoplasmic onion in Indian populations. The genetics of male fertility restoration for $\mathrm{S}$ cytoplasm were studied by Jones and Clarke (1943) using crosses of the original source of S cytoplasm (Italian Red 13-53) with a few North American populations. Onion was likely domesticated in Central Asia and spread in a western direction toward the Mediterranean region, through Europe and North Africa, to North and South America, and ultimately from North America to Japan and East Asia (Goldman et al., 2000). Onion also spread in a south-eastern direction from Central Asia into the Indian subcontinent. As a result, onion germplasm commonly grown in East Asia may be of European origin and share the same male fertility restoration locus for $\mathrm{S}$ cytoplasm as European and American (Northern and Southern) germplasm, as evidenced by the effectiveness of jnurf13 and AcPms1 to predict genotypes at $M s$ for North American (this research) and East Asian (Kim et al.,
2015) onions. Robust cytoplasmic [such as the accD polymorphism (Khar and Saini, 2016; von Kohn et al., 2013)] and nuclear (AcPms1) markers should help to resolve inconsistencies regarding the genetics of male fertility restoration across different onion populations.

\section{Literature Cited}

Bang, H., S. Kim, S.O. Park, K. Yoo, and B.S. Patil 2013. Development of a codominant CAPS marker linked to the $M s$ locus controlling fertility restoration in onion (Allium cepa $\mathrm{L}$.). Sci. Hort. 153:42-49.

Gökçe, A.F. and M.J. Havey. 2002. Linkage equilibrium among tightly linked RFLPs and the $M s$ locus in open-pollinated onion populations. J. Amer. Soc. Hort. Sci. 127:944-946.

Gökçe, A.F., J. McCallum, Y. Sato, and M.J. Havey. 2002. Molecular tagging of the Ms locus in onion. J. Amer. Soc. Hort. Sci. 127:576-582.

Goldman, I.L., G. Schroeck, and M.J. Havey. 2000. History of public onion breeding programs and pedigree of public onion germplasm releases in the United States. Plant Breed. Rev. 20:67-103.

Havey, M.J. 1993. A putative donor of S-cytoplasm and its distribution among open-pollinated populations of onion. Theor. Appl. Genet. 86:128-134.

Havey, M.J. 1995. Identification of cytoplasms using the polymerase chain reaction to aid in the extraction of maintainer lines from openpollinated populations of onion. Theor. Appl. Genet. 90:263-268.

Havey, M.J. 2013. Single nucleotide polymorphisms in linkage disequilibrium with the male-sterility restoration $(M s)$ locus in openpollinated and inbred populations of onion. J. Amer. Soc. Hort. Sci. 138:306-309.

Huo, Y.M., J. Miao, B.J. Liu, Y.Y. Yang, Y.H. Zhang, and X. Wu. 2012. The expression of pectin methylesterase in onion flower buds is associated with the dominant male-fertility restoration allele. Plant Breed. 131:211-216.

Jones, H.A. and A. Clarke. 1943. Inheritance of male sterility in the onion and the production of hybrid seed. Proc. Amer. Soc. Hort. Sci. 43:189194.

Khar, A. and N. Saini. 2016. Limitations of PCRbased molecular markers to identify malesterile and maintainer plants from Indian onion (Allium cepa L.) populations. Plant Breed. 135:519-524.

Khrustaleva, L., J. Jiang, and M.J. Havey. 2016. High-resolution tyramide-FISH mapping of markers tightly linked to the male-fertility restoration (Ms) locus of onion. Theor. Appl. Genet. 129:535-545.

Kim, S. 2014. A codominant molecular marker in linkage disequilibrium with a restorer-offertility gene $(M s)$ and its application in reevaluation of inheritance of fertility restoration in onions. Mol. Breed. 34:769-778.

Kim, S., C.-W. Kim, M. Park, and D. Choi. 2015. Identification of candidate genes associated with fertility restoration of cytoplasmic malesterility in onion (Allium cepa L.) using a combination of bulked segregant analysis and RNA-seq. Theor. Appl. Genet. 128:22892299.

Kim, S., E. Lee, D.Y. Cho, T. Han, H. Bang, B.S. Patil, Y.K. Ahn, and M. Yoon. 2009. Identification of a novel chimeric gene, orf 725 , and its use in development of a molecular marker for distinguishing three cytoplasm types in onion (Allium cepa L.). Theor. Appl. Genet. 118:433441.

Park, J., H. Bang, D.Y. Cho, M. Yoon, B.S. Patil, and S. Kim. 2013. Construction of highresolution linkage map of the $M s$ locus, a restorer-of-fertility gene in onion (Allium cepa L.). Euphytica 192:267-278.

Sato, Y. 1998. PCR amplification of CMS-specific mitochondrial nucleotide sequences to identify cytoplasmic genotypes of onion (Allium cepa L.). Theor. Appl. Genet. 96:367-370.

von Kohn, C., A. Kiełkowska, and M.J. Havey. 2013. Sequencing and annotation of the chloroplast DNAs of normal $(\mathrm{N})$ male-fertile and male-sterile (S) cytoplasms of onion and single nucleotide polymorphisms distinguishing these cytoplasms. Genome 56:737742.

Yang, Y., Y. Huo, J. Miao, B. Liu, S. Kong, L. Gao, C. Liu, Z. Wang, Y. Tahara, H. Kitano, and X. Wu. 2012. Identification of two SCAR markers co-segregated with the dominant $M s$ and recessive ms alleles in onion (Allium cepa $\mathrm{L}$.). Euphytica 190:267-277. 